\title{
Electronically Controllable Sinusoidal Oscillator Employing CMOS VD-DIBAs
}

\author{
Dinesh Prasad, ${ }^{1}$ D. R. Bhaskar, ${ }^{1}$ and K. L. Pushkar ${ }^{2}$ \\ ${ }^{1}$ Department of Electronics and Communication Engineering, Faculty of Engineering and Technology, Jamia Millia Islamia, \\ New Delhi 110025, India \\ ${ }^{2}$ Department of Electronics and Communication Engineering, Maharaja Agrasen Institute of Technology, Rohini, \\ New Delhi 110058, India
}

Correspondence should be addressed to Dinesh Prasad; dprasad@jmi.ac.in

Received 23 November 2012; Accepted 19 December 2012

Academic Editors: L.-F. Mao, E. Tlelo-Cuautle, and Z.-M. Tsai

Copyright (C) 2013 Dinesh Prasad et al. This is an open access article distributed under the Creative Commons Attribution License, which permits unrestricted use, distribution, and reproduction in any medium, provided the original work is properly cited.

\begin{abstract}
A new electronically controllable sinusoidal oscillator employing two voltage differencing-differential input buffered amplifiers (VD-DIBAs), two grounded capacitors, and one grounded resistor is presented. The proposed configuration offers (i) independent control of condition of oscillation (CO) and frequency of oscillation (FO) formerly by resistance and later through transconductance, (ii) low active and passive sensitivities, and (iii) a good frequency stability. The workability of the proposed configuration has been demonstrated by SPICE simulation.
\end{abstract}

\section{Introduction}

Sinusoidal oscillators find numerous applications in communication, control systems, signal processing, instrumentation, and measurement systems. In the recent past, a large number of single resistance controlled oscillators (SRCOs) have been proposed, see [1-11]; however, in all these SRCOs, the frequency of oscillation can be controlled by varying the values of resistances involved. Obviously, by replacing one of the grounded resistors by JFETs/MOSFETs, electronic tunability can be established, see $[2,4]$ and the references cited therein. Electronically controllable sinusoidal oscillators (ECSOs) based on different active building blocks are available in the literature, see [12-17] and the references cited therein. The advantages, applications, and usefulness of recently introduced new active building block named voltage differencing-differential input buffered amplifier (VD-DIBA) are now being recognized in the literature [18-20]. Recently, electronically controllable grounded and floating simulated inductance circuits using VD-DIBAs have been introduced in [20]. However, to the best knowledge and belief of the authors, no ECSO using VD-DIBAs has yet been presented in the open literature so far. The purpose of this paper is, therefore, to propose a new ECSO using VD-DIBAs along with a minimum possible number of grounded passive components, which offers (i) independent control of oscillation frequency and condition of oscillation, (ii) low active and passive sensitivities, and (iii) a good frequency stability factor.

\section{The Proposed Configuration}

The schematic symbol and behavioral model of the VDDIBA are shown in Figures 1(a) and 1(b), respectively [18]. The model includes two controlled sources: the current source controlled by differential voltage $\left(V_{+}-V_{-}\right)$, with the transconductance $g_{m}$, and the voltage source controlled by differential voltage $\left(V_{z}-V_{v}\right)$, with the unity voltage gain. The VD-DIBA can be described by the following set of equations:

$$
\left(\begin{array}{c}
I_{+} \\
I_{-} \\
I_{z} \\
I_{v} \\
V_{w}
\end{array}\right)=\left(\begin{array}{ccccc}
0 & 0 & 0 & 0 & 0 \\
0 & 0 & 0 & 0 & 0 \\
g_{m} & -g_{m} & 0 & 0 & 0 \\
0 & 0 & 0 & 0 & 0 \\
0 & 0 & 1 & -1 & 0
\end{array}\right)\left(\begin{array}{c}
V_{+} \\
V_{-} \\
V_{z} \\
V_{v} \\
I_{w}
\end{array}\right) \text {. }
$$

The proposed new ECSO configuration is shown in Figure 2. 


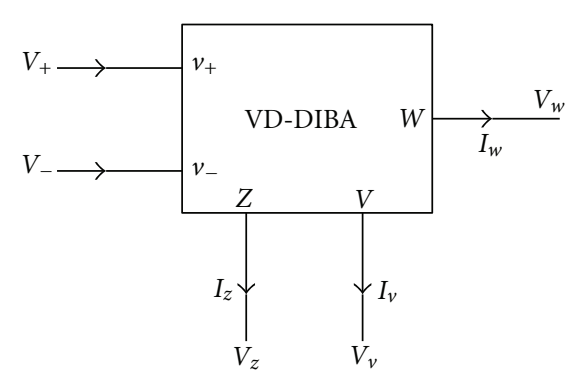

(a)

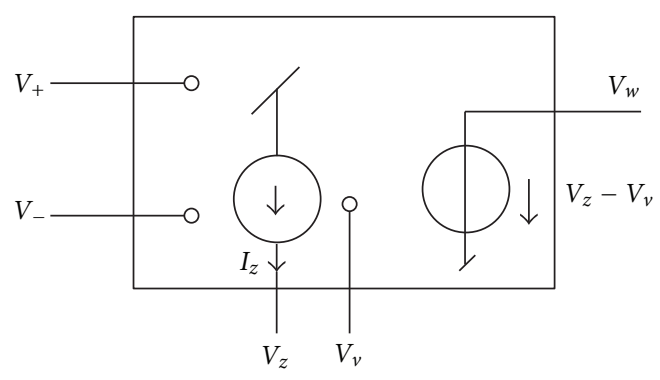

(b)

FIgURE 1: (a) Schematic symbol, (b) behavioral model of VD-DIBA.

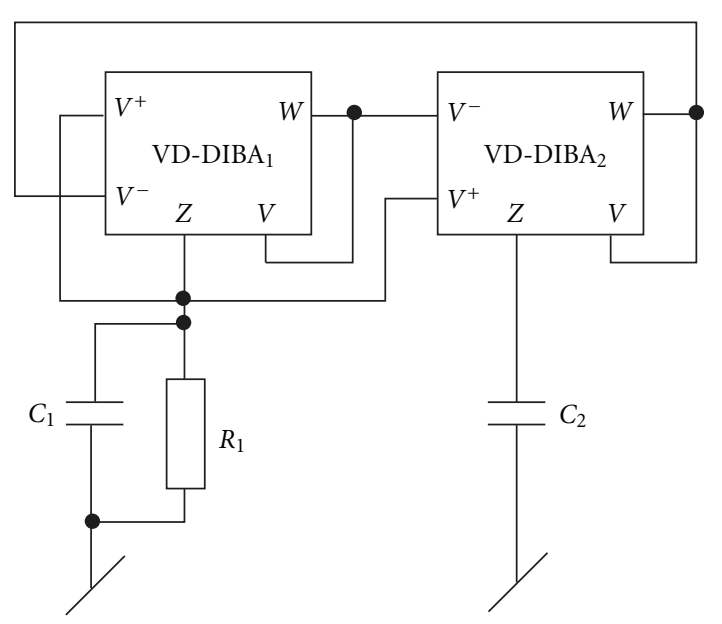

FIGURE 2: The proposed configuration.

TABLE 1

\begin{tabular}{lc}
\hline Transistor & $W / L(\mu \mathrm{m})$ \\
\hline M1, M2, M5, M6, M9, M10, M13-M18 & $3.6 / 0.36$ \\
M3, M4, M7, M8, M11, M12, M19-M24 & $16.6 / 0.36$ \\
\hline
\end{tabular}

A routine circuit analysis of Figure 2 yields the following characteristic equation:

$$
s^{2}+s \frac{1}{C_{1}}\left(\frac{1}{R_{1}}-g_{m_{1}}\right)+\frac{g_{m_{1}} g_{m_{2}}}{4 C_{1} C_{2}}=0 .
$$

Thus, the condition of oscillation (CO) and frequency of oscillation (FO) are given by

$$
\begin{gathered}
\left(\frac{1}{R_{1}}-g_{m_{1}}\right) \leq 0, \\
\omega_{0}=\sqrt{\frac{g_{m_{1}} g_{m_{2}}}{4 C_{1} C_{2}} .}
\end{gathered}
$$

Therefore, it is seen that FO is independently controllable by transconductance $g_{m_{2}}$ of the VD-DIBA 2 (which is current controllable by bias current, $I_{\mathrm{B} 2}$ ), whereas $\mathrm{CO}$ is independently established through the resistor $R_{1}$. The above routine circuit analysis can also be obtained using the analysis performance as given in $[21,22]$.
The CMOS implementation of VD-DIBA is shown in Figure 3. For this purpose, the TSMC CMOS $0.18 \mu \mathrm{m}$ process parameters are used for all MOSFETs in the circuit of Figure 3.

Transistor aspect ratios are indicated in Table 1.

The TSMC CMOS $0.18 \mu \mathrm{m}$ process parameters are given in Table 2.

\section{Nonideal Analysis}

Let $R_{z}$ and $C_{z}$ denote the parasitic resistance and parasitic capacitance of the $Z$ terminal. Taking into account the nonidealities of the VD-DIBA, namely $V_{w}=\left(\beta^{+} V_{z}-\beta^{-} V_{v}\right)$, where $\beta^{+}=1-\varepsilon_{1}\left(\varepsilon_{1} \ll 1\right)$ and $\beta^{-}=1-\varepsilon_{2}\left(\varepsilon_{2} \ll 1\right)$ are voltage tracking errors of the VD-DIBA then the expressions for $\mathrm{CO}$ and $\mathrm{FO}$ are found to be

$$
\left\{\left(C_{2}+C_{Z}\right)\left(\frac{1}{R_{1}}-g_{m 1}\right)+\frac{1}{R_{Z}}\left(C_{1}+C_{2}+2 C_{Z}\right)\right\} \leq 0,
$$

$$
\omega_{0}=\sqrt{\frac{\left\{\frac{\left(1 / R_{1}+1 / R_{Z}-g_{m_{1}}\right)}{R_{Z}}+\frac{\beta_{2}^{+}\left(1+\beta_{1}^{-}-\beta_{1}^{+}\right) g_{m_{1}} g_{m_{2}}}{\left(1+\beta_{2}^{-}\right)\left(1+\beta_{1}^{-}\right)}\right\}}{C_{1} C_{2}} .}
$$

Taking $C_{1}=C_{2}=C \gg C_{z}$, and $R_{z} \gg R_{1}$, then (4) reduces to

$$
\begin{gathered}
\left(\frac{1}{R_{1}}-g_{m_{1}}\right) \leq 0, \\
\omega_{0}=\sqrt{\frac{\left(1+\beta_{1}^{-}-\beta_{1}^{+}\right) \beta_{2}^{+} g_{m_{1}} g_{m_{2}}}{\left(1+\beta_{1}^{-}\right)\left(1+\beta_{2}^{-}\right) C^{2}} .}
\end{gathered}
$$

This nonideal analysis can also be determined using the analysis performance as given in [23].

The active and passive sensitivities are calculated as

$$
\begin{array}{r}
S_{C}^{\omega_{0}}=-1, \quad S_{\beta_{1}^{+}}^{\omega_{0}}=-\frac{\beta_{1}^{+}}{2\left(1+\beta_{1}^{-}-\beta_{1}^{+}\right)}, \\
S_{\beta_{1}^{-}}^{\omega_{0}}=\frac{\beta_{1}^{+} \beta_{1}^{-}}{2\left(1+\beta_{1}^{-}-\beta_{1}^{+}\right)\left(1+\beta_{1}^{-}\right)}, \quad S_{\beta_{2}^{+}}^{\omega_{0}}=\frac{1}{2},
\end{array}
$$




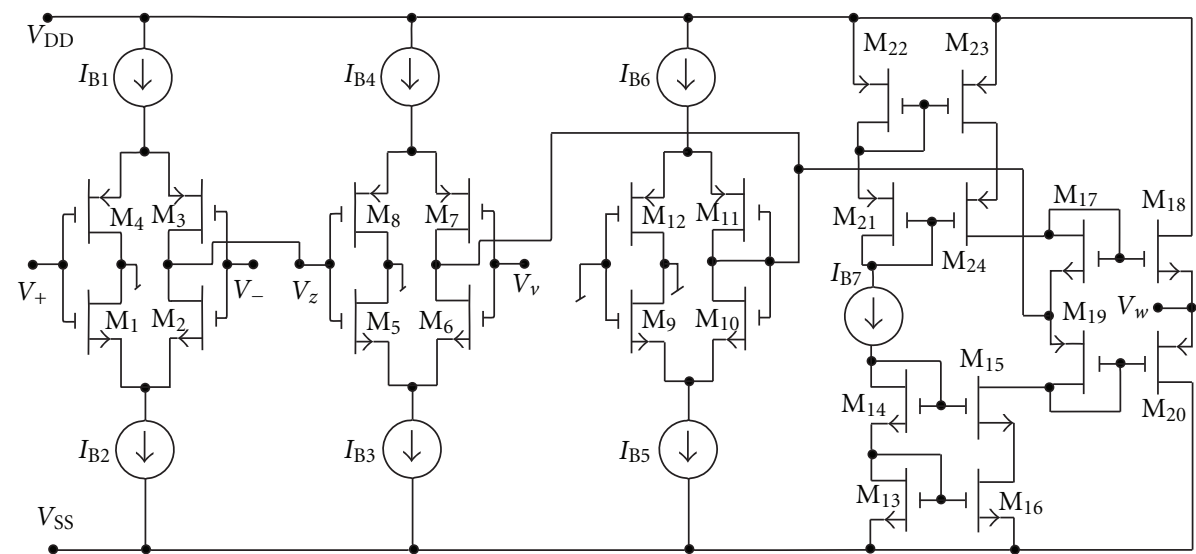

FIGURE 3: CMOS Implementation of VD-DIBA, $V_{\mathrm{DD}}=-V_{\mathrm{SS}}=1 \mathrm{~V}, I_{\mathrm{B} 1}=I_{\mathrm{B} 2}=I_{\mathrm{B} 3}=I_{\mathrm{B} 4}=I_{\mathrm{B} 5}=I_{\mathrm{B} 6}=150 \mu \mathrm{A}$ and $I_{\mathrm{B} 7}=30 \mu \mathrm{A}[20]$.

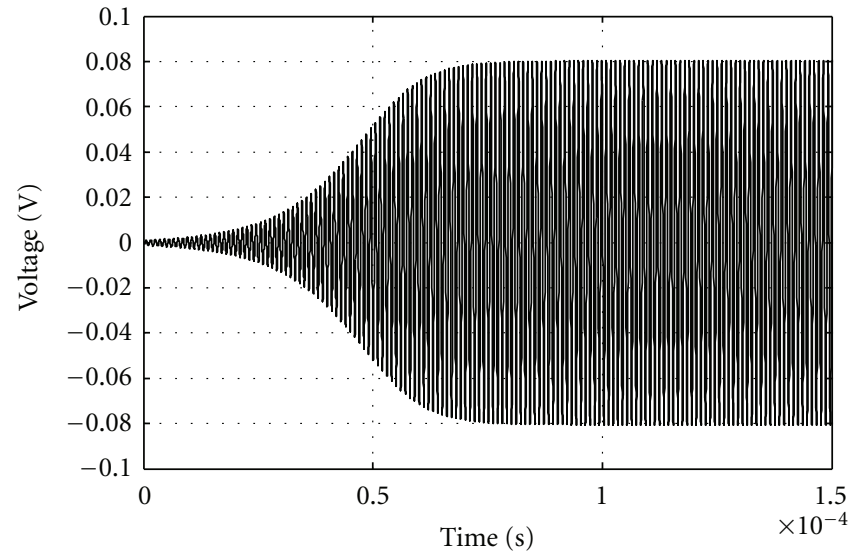

(a)

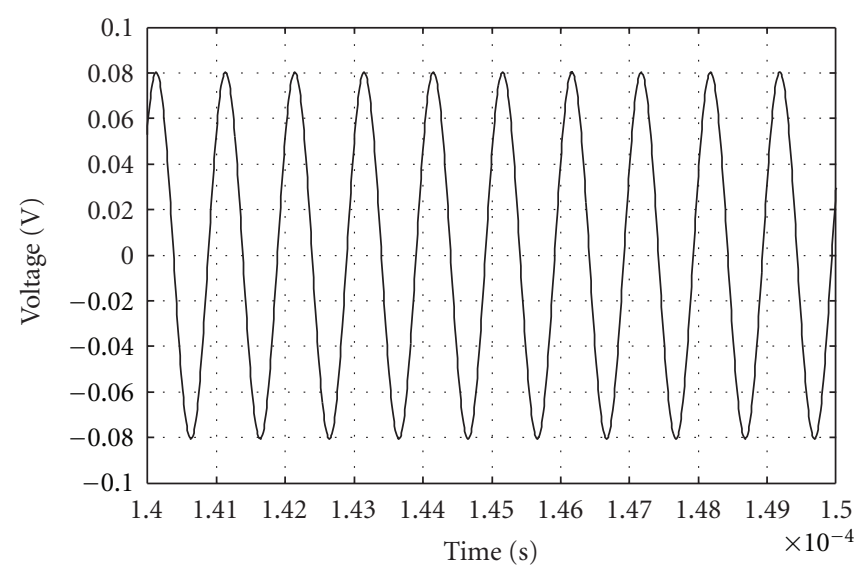

(b)

Figure 4: (a) Transient output waveform, (b) steady-state response of the output.

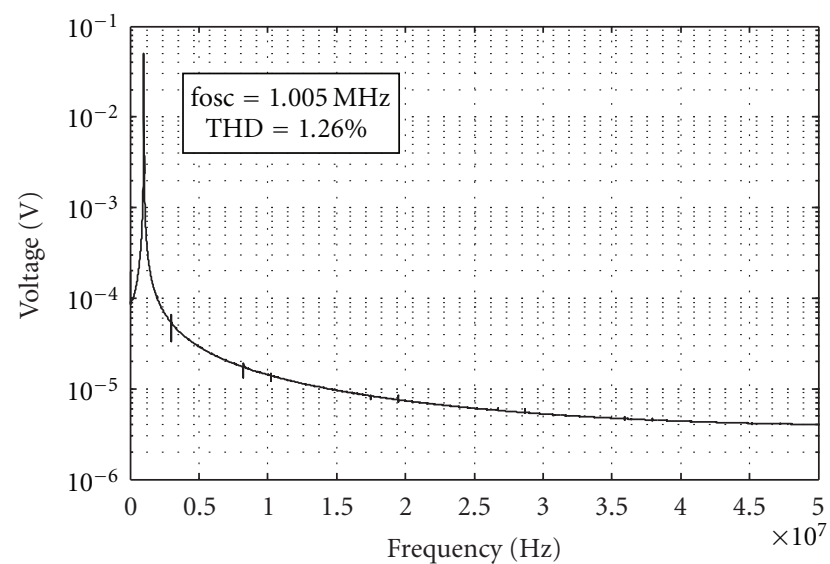

FIGURE 5: Simulation result of the output spectrum.

$$
S_{\beta_{2}^{-}}^{\omega_{0}}=-\frac{\beta_{2}^{-}}{2\left(1+\beta_{2}^{-}\right)}, \quad S_{g_{m_{1}}}^{\omega_{0}}=\frac{1}{2}, \quad S_{g_{m_{2}}}^{\omega_{0}}=\frac{1}{2},
$$

which are all low.

\section{Frequency Stability}

Using the definition of the frequency stability factor $S^{F}$ as given in [2-4] $S^{F}=\left.(d \phi(u) / d u)\right|_{u=1}$ (where $u=\omega / \omega_{0}$ is the normalized frequency and $\phi(u)$ represents the phase of the open-loop transfer function of the oscillator circuit), with $C_{1}=C_{2}=C, g_{m_{1}}=1 / R_{1}=g_{m}$ and $g_{m_{2}}=n g_{m}$, where $n$ is a frequency-controlling transconductor ratio, the $S^{F}$ of the proposed oscillator is found to be $\sqrt{n}$. Therefore, a good frequency stability is obtainable by selecting larger value of $n$.

\section{Simulation Results}

To verify the theoretical analysis, the proposed circuit has been simulated using the CMOS-based VD-DIBA (Figure 3). The component values used were $C_{1}=C_{2}=0.05 \mathrm{nF}$, and $R_{1}=1.65 \mathrm{~K} \Omega$, the CMOS VD-DIBA was biased with $\pm 1 \mathrm{~V}$ D.C. power supplies with $I_{\mathrm{B} 1}=I_{\mathrm{B} 2}=I_{\mathrm{B} 3}=I_{\mathrm{B} 4}=$ $I_{\mathrm{B} 5}=I_{\mathrm{B} 6}=150 \mu \mathrm{A}$ and $I_{\mathrm{B} 7}=30 \mu \mathrm{A}$. The transconductances of VD-DIBA are controlled by bias currents. SPICE generated output waveforms indicating transient and steady state responses are shown in Figures 4(a) and 4(b), respectively. 


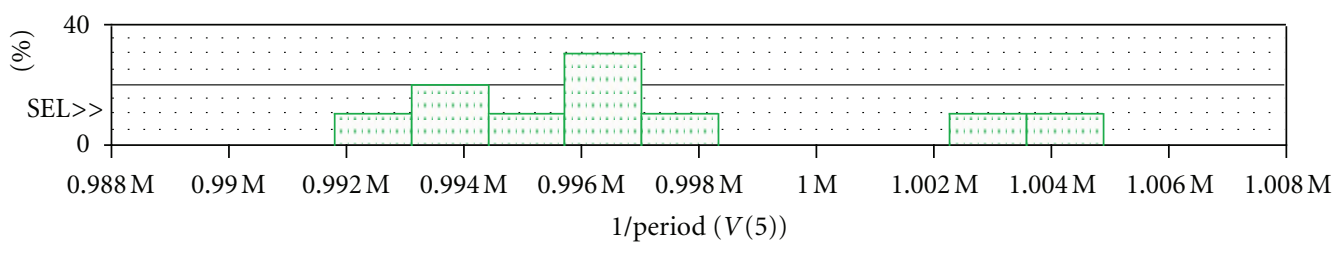

\begin{tabular}{|llll|}
\hline$n$ samples $=10$ & Sigma $=4113.45$ & Median $=995983$ & 3* sigma $=12340.4$ \\
$n$ divisions $=10$ & Minimum $=991797$ & 90th \%ile $=1.00384 e+006$ & \\
Mean $=996794$ & 10th \%ile $=992577$ & Maximum $=1.00488 e+006$ & \\
\hline
\end{tabular}

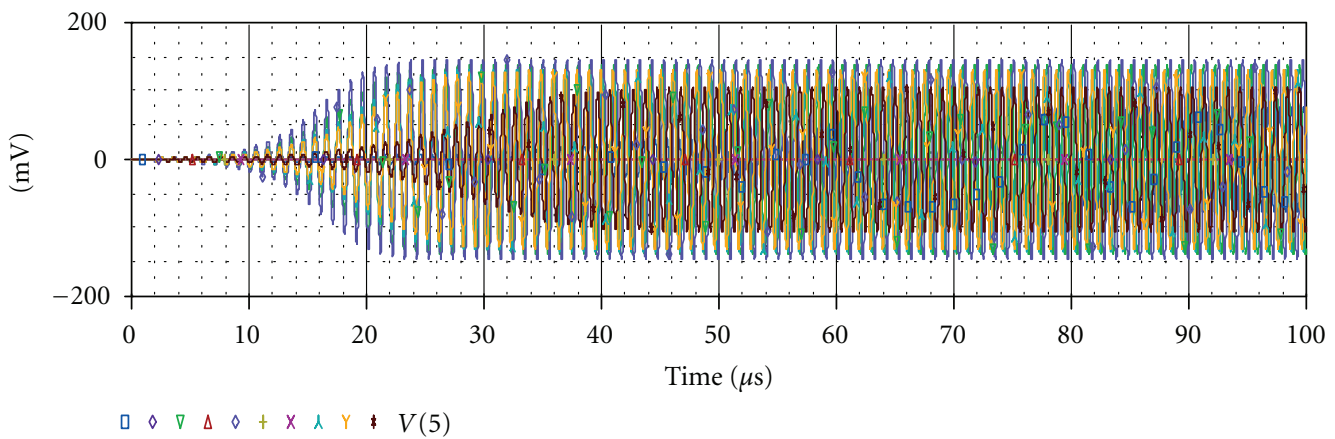

Figure 6: Result of Monte-Carlo Simulation of oscillator circuit of Figure 2.

TABle 2

\begin{tabular}{|c|c|}
\hline MODEL $n$ nmos & MODEL $p$ pmos \\
\hline $\begin{array}{l}\mathrm{LEVEL}=7, \mathrm{VERSION}=3.1 \mathrm{TNOM}=27 \mathrm{TOX}=4.1 E-9 \mathrm{XJ}= \\
1 E-7 \mathrm{NCH}=2.3549 E 17 \mathrm{VTH} 0=0.3696986 \mathrm{~K} 1=0.6064385 \mathrm{~K} 2= \\
1.63871 E-3 \mathrm{~K} 3=1 E-3, \mathrm{~K} 3 \mathrm{~B}=2.763267 \mathrm{~W} 0=1 E-7 \mathrm{NLX}= \\
1.71872 E-7 \mathrm{DVT} 0 \mathrm{~W}=0 \mathrm{DVT} 1 \mathrm{~W}=0 \mathrm{DVT} 2 \mathrm{~W}=0, \mathrm{DVT} 0= \\
1.3330881 \mathrm{DVT} 1=0.3683763 \mathrm{DVT} 2=0.0540199 \mathrm{U} 0=258.9066683 \\
\mathrm{UA}=-1.504141 E-9 \mathrm{UB}=2.428646 E-18 \mathrm{UC}=5.105195 E-11 \\
\mathrm{VSAT}=9.896282 E 4 \mathrm{~A} 0=1.8904342 \mathrm{AGS}=0.4044483 \mathrm{~B} 0= \\
-4.706134 E-8 \mathrm{~B} 1=1.294942 E-6 \mathrm{KETA}=-2.730673 E-3 \mathrm{~A} 1= \\
5.916677 E-4 \mathrm{~A} 2=0.9069159 \mathrm{RDSW}=105 \mathrm{PRWG}=0.5 \mathrm{PRWB}= \\
-0.2 \mathrm{WR}=1 \mathrm{WINT}=0 \mathrm{LINT}=1.69494 E-8, \mathrm{XL}=0 \mathrm{XW}=-1 E-8 \\
\mathrm{DWG}=-3.773529 E-9 \mathrm{DWB}=5.239518 E-9 \mathrm{VOFF}=-0.0883818 \\
\mathrm{NFACTOR}=2.1821266 \mathrm{CIT}=0 \mathrm{CDSC}=2.4 E-4 \mathrm{CDSCD}=0, \\
\mathrm{CDSCB}=0 \mathrm{ETA} 0=2.470369 E-3 \mathrm{ETAB}=1.047744 E-5 \mathrm{DSUB}= \\
0.0167866 \mathrm{PCLM}=0.7326932 \mathrm{PDIBLC} 1=0.1823102, \mathrm{PDIBLC} 2= \\
3.38377 E-3 \mathrm{PDIBLCB}=-0.1 \mathrm{DROUT}=0.7469045 \mathrm{PSCBE} 1= \\
8 E 10 \mathrm{PSCB} 2=1.254966 E-9 \mathrm{PVAG}=0, \mathrm{DELTA}=0.01 \mathrm{RSH}=6.5 \\
\mathrm{MOBMOD}=1 \mathrm{PRT}=0 \mathrm{UTE}=-1.5 \mathrm{KT} 1=-0.11 \mathrm{KT} 1 \mathrm{~L}=0 \mathrm{KT} 2= \\
0.022 \mathrm{UA} 1=4.31 E-9 \mathrm{UB} 1=-7.61 E-18 \mathrm{UC} 1=-5.6 E-11 \mathrm{AT}= \\
3.3 E 4 \mathrm{WL}=0 \mathrm{WLN}=1 \mathrm{WW}=0 \mathrm{WWN}=1 \mathrm{WWL}=0 \mathrm{LL}=0 \mathrm{LLN} \\
=1 \mathrm{LW}=0 \mathrm{LWN}=1 \mathrm{LWL}=0 \mathrm{CAPMOD}=2 \mathrm{XPART}=0.5 \mathrm{CGDO} \\
=7.9 E-10 \mathrm{CGSO}=7.9 E-10 \mathrm{CGBO}=1 E-12 \mathrm{CJ}=9.539798 E-4 \\
\mathrm{~PB}=0.8 \mathrm{MJ}=0.380768 \mathrm{CJSW}=2.53972 E-10 \mathrm{PBSW}=0.8 \mathrm{MJSW} \\
=0.1061193 \mathrm{CJSWG}=3.3 E-10 \mathrm{PBSWG}=0.8 \mathrm{MJSWG}=0.1061193 \\
\mathrm{CF}=0 \mathrm{PVTH}=7.505753 E-4 \mathrm{PRDSW}=-2.7650517 \mathrm{PK} 2= \\
-4.42044 E-4 \mathrm{WKETA}=3.100384 E-3 \mathrm{LKETA}=-0.0104103 \mathrm{PU} 0 \\
=10.8203648 \mathrm{PUA}=2.896652 E-11 \mathrm{PUB}=1.684125 E-23 \mathrm{PVSAT} \\
=1.388017 E 3 \mathrm{PETA} 0=8.758549 E-5 \mathrm{PKETA}=1.549791 E-3\end{array}$ & $\begin{array}{l}\text { LEVEL }=7 \mathrm{VERSION}=3.1 \mathrm{TNOM}=27 \mathrm{TOX}=4.1 E-9 \mathrm{XJ}= \\
1 E-7 \mathrm{NCH}=4.1589 E 17 \mathrm{VTH} 0=-0.3835898 \mathrm{~K} 1=0.59111 \mathrm{~K} 2= \\
0.0258663 \mathrm{~K} 3=0 \mathrm{~K} 3 \mathrm{~B}=7.9143108 \mathrm{~W} 0=1 E-6 \mathrm{NLX}= \\
1.20187 E-7 \mathrm{DVT} 0 \mathrm{~W}=0 \mathrm{DVT} 1 \mathrm{~W}=0 \mathrm{DVT} 2 \mathrm{~W}=0 \mathrm{DVT} 0= \\
0.6117215 \mathrm{DVT} 1=0.2286816 \mathrm{DVT} 2=0.1 \mathrm{U} 0=106.5280265 \mathrm{UA}= \\
1.125454 E-9 \mathrm{UB}=1 E-21 \mathrm{UC}=-1 E-10 \mathrm{VSAT}=1.593712 E 5 \\
\mathrm{~A} 0=1.6904754 \mathrm{AGS}=0.3667554 \mathrm{~B} 0=5.263128 E-7 \mathrm{~B} 1= \\
1.496707 E-6 \mathrm{KETA}=0.0237092 \mathrm{~A} 1=0.2276342 \mathrm{~A} 2=0.6915706 \\
\mathrm{RDSW}=304.9893888 \mathrm{PRWG}=0.5 \mathrm{PRWB}=0.2553725 \mathrm{WR}=1 \\
\mathrm{WINT}=0 \mathrm{LINT}=3.217673 E-8 \mathrm{XL}=0 \mathrm{XW}=-1 E-8 \mathrm{DWG}= \\
-2.44019 E-8 \mathrm{DWB}=-9.06003 E-10 \mathrm{VOFF}=-0.0878287 \\
\text { NFACTOR }=1.8560303 \mathrm{CIT}=0 \mathrm{CDSC}=2.4 E-4 \mathrm{CDSCD}=0 \\
\mathrm{CDSCB}=0 \mathrm{ETA} 0=0.1672562 \mathrm{ETAB}=-0.1249603 \mathrm{DSUB}= \\
1.0998181 \mathrm{PCLM}=2.2249148 \mathrm{PDIBLC} 1=8.275696 E-4 \\
\mathrm{PDIBLC}=0.0420477 \mathrm{PDIBLCB}=-1 E-3 \mathrm{DROUT}=0 \mathrm{PSCBE} 1 \\
=1.073111 E 10 \mathrm{PSCBE} 2=3.099395 E-9 \mathrm{PVAG}=15 \mathrm{DELTA}= \\
0.01 \mathrm{RSH}=7.4 \mathrm{MOBMOD}=1 \mathrm{PRT}=0 \mathrm{UTE}=-1.5 \mathrm{KT} 1=-0.11 \\
\mathrm{KT} 1 \mathrm{~L}=0 \mathrm{KT} 2=0.022 \mathrm{UA} 1=4.31 E-9+\mathrm{UB} 1=-7.61 E-18 \mathrm{UC} 1 \\
=-5.6 E-11 \mathrm{AT}=3.3 E 4 \mathrm{WL}=0 \mathrm{WLN}=1 \mathrm{WW}=0 \mathrm{WWN}=1 \\
\mathrm{WWL}=0 \mathrm{LL}=0 \mathrm{LLN}=1 \mathrm{LW}=0 \mathrm{LWN}=1 \mathrm{LWL}=0 \mathrm{CAPMOD}= \\
2 \mathrm{XPART}=0.5 \mathrm{CGDO}=6.41 E-10 \mathrm{CGSO}=6.41 E-10 \mathrm{CGBO}= \\
1 E-12 \mathrm{CJ}=1.200422 E-3 \mathrm{~PB}=0.8478616 \mathrm{MJ}=0.4105254 \mathrm{CJSW} \\
=2.001802 E-10 \mathrm{PBSW}=0.8483594 \mathrm{MJSW}=0.3400571 \mathrm{CJSWG} \\
=4.22 E-10 \mathrm{PBSWG}=0.8483594 \mathrm{MJSWG}=0.3400571 \mathrm{CF}=0 \\
\mathrm{PVTH}=2.098588 E-3 \mathrm{PRDSW}=4.4771801 \mathrm{PK} 2= \\
1.799383 E-3 \mathrm{WKETA}=0.0295614 \mathrm{LKETA}=-1.935751 E-3 \\
\mathrm{PU} 0=-1.3399122 \mathrm{PUA}=-5.27759 E-11 \mathrm{PUB}=1 E-21 \mathrm{PVSAT} \\
=-50 \mathrm{PETA} 0=1.003159 E-4 \mathrm{PKETA}=-3.434535 E-3\end{array}$ \\
\hline
\end{tabular}


TABLE 3

\begin{tabular}{lcccc}
\hline $\begin{array}{l}\text { Reference } \\
\text { number }\end{array}$ & $\begin{array}{c}\text { No. of } \\
\text { active } \\
\text { elements }\end{array}$ & $\begin{array}{c}\text { No. of } \\
\text { passive } \\
\text { elements }\end{array}$ & $\begin{array}{c}\text { No. of } \\
\text { grounded } \\
\text { capacitors }\end{array}$ & $\begin{array}{c}\text { Independent } \\
\text { electronic } \\
\text { tunability }\end{array}$ \\
\hline$[12]$ & 2 & 3 to 7 & 2 & Yes \\
{$[13]$} & 3 & 2 & 2 & Yes \\
{$[14]$} & 3 & 3 & 3 & Yes \\
{$[15]$} & 3 & 2 & 2 & Yes \\
{$[16]$} & 4 & 2 & 1 & No \\
Proposed & 2 & 3 & 2 & Yes \\
\hline
\end{tabular}

These results, thus, confirm the validity of the proposed configuration. Figure 5 shows the output spectrum, where the total harmonic distortion (THD) is found to be $1.26 \%$. The oscillator circuit of Figure 2 has been checked for robustness using Monte-Carlo simulations, the sample result has been shown in Figure 6, which confirms that for $\pm 10 \%$ variations in the value of $R_{1}$, the value of oscillation frequency remain close to its normal value of $1.005 \mathrm{MHz}$ and hence almost unaffected by change in $R_{1}$. A comparison with other previously known ECSOs has been given in Table 3 .

\section{Concluding Remarks}

A new circuit configuration employing two VD-DIBAs along with a minimum possible number of grounded passive elements (i.e., only one resistor and two capacitors) has been proposed. In oscillator mode, the circuit offers (i) independent control of condition of oscillation and frequency of oscillation former by resistance and later through transconductance, (ii) low active and passive sensitivities, and (iii) a good frequency stability for larger values of $n$. The validity of the proposed circuit has been established by SPICE simulations.

\section{References}

[1] R. Senani, "New types of sine wave oscillators," IEEE Transactions on Instrumentation and Measurement, vol. 34, no. 3, pp. 461-463, 1985.

[2] D. R. Bhaskar and R. Senani, "New CFOA-based singleelement-controlled sinusoidal oscillators," IEEE Transactions on Instrumentation and Measurement, vol. 55, no. 6, pp. 2014-2021, 2006.

[3] D. R. Bhaskar and R. Senani, "New current-conveyor-based single-resistance-controlled/voltage-controlled oscillator employing grounded capacitors," Electronics Letters, vol. 29, no. 7, pp. 612-614, 1993.

[4] S. S. Gupta and R. Senani, "Realisation of current-mode SRCOs using all grounded passive elements," Frequenz, vol. 57, no. 1-2, pp. 25-36, 2003.

[5] V. Kumar, K. Pal, and G. K. Gupta, "Novel single resistance controlled sinusoidal oscillator using FTFN and OTA," Indian Journal of Pure and Applied Physics, vol. 44, no. 8, pp. 625-627, 2006.
[6] J. W. Horng, S. F. Lin, and C. T. Yang, "Sinusoidal oscillators using current conveyors and grounded capacitors," Journal of Active and Passive Electronic Devices, vol. 2, pp. 127-136, 2007.

[7] S. I. Liu, "Single-resistance-controlled sinusoidal oscillator using two FTFNs," Electronics Letters, vol. 33, no. 14, pp. 1185-1186, 1997.

[8] Soliman and A. M, "Current mode CCII oscillators using grounded capacitors and resistors," International Journal of Circuit Theory and Applications, vol. 26, pp. 431-438, 1998.

[9] S. S. Gupta, R. K. Sharma, D. R. Bhaskar, and R. Senani, "Sinusoidal oscillators with explicit current output employing current-feedback op-amps," International Journal of Circuit Theory and Applications, vol. 38, no. 2, pp. 131-147, 2010.

[10] H. A. Alzaher, "CMOS digitally programmable quadrature oscillators," International Journal of Circuit Theory and Applications, vol. 36, no. 8, pp. 953-966, 2008.

[11] E. Tlelo-Cuautle, M. A. Duarte-Villaseñor, J. M. García-Ortega, and C. Sánchez-López, "Designing SRCOs by combining SPICE and Verilog-A," International Journal of Electronics, vol. 94, no. 4, pp. 373-379, 2007.

[12] Y. Tao and J. Kel Fidler, "Electronically tunable dual-OTA second-order sinusoidal oscillators/filters with non-interacting controls: a systematic synthesis approach," IEEE Transactions on Circuits and Systems I: Fundamental Theory and Applications, vol. 47, no. 2, pp. 117-129, 2000.

[13] D. R. Bhaskar, M. P. Tripathi, and R. Senani, "Systematic derivation of all possible canonic OTA-C sinusoidal oscillators," Journal of the Franklin Institute, vol. 330, no. 5, pp. 885-903, 1993.

[14] T. Tsukutani, Y. Sumi, and Y. Fukui, "Electronically controlled current-mode oscillators using MO-OTAs and grounded capacitors," Frequenz, vol. 60, no. 11-12, pp. 220-223, 2006.

[15] D. R. Bhaskar, K. K. Abdalla, and R. Senani, "Electronicallycontrolled current-mode second order sinusoidal oscillators using MO-OTAs and grounded capacitors," Circuits and Systems, vol. 2, no. 1, pp. 65-73, 2011.

[16] M. T. Abuelma'atti, "A new electronically tunable integrable CCII-OTA-based active-C oscillator," European Transactions on Telecommunications, vol. 2, no. 3, pp. 353-355, 1991.

[17] G. Souliotis and C. Psychalinos, "Electronically controlled multiphase sinusoidal oscillators using current amplifiers," International Journal of Circuit Theory and Applications, vol. 37, no. 1, pp. 43-52, 2009.

[18] D. Biolek, R. Senani, V. Biolkova, and Z. Kolka, "Active elements for analog signal processing: classification, review, and new proposals," Radioengineering, vol. 17, no. 4, pp. 15-32, 2008.

[19] D. Biolek and V. Biolkova, "First-order voltage-mode allpass filter employing one active element and one grounded capacitor," Analog Integrated Circuits and Signal Processing, vol. 65, no. 1, pp. 123-129, 2010.

[20] D. Prasad, D. R. Bhaskar, and K. L. Pushkar, "Realization of new electronically controllable grounded and floating simulated inductance circuits using voltage differencing differential input buffered amplifiers," Active and Passive Electronic Components, vol. 2011, Article ID 101432, 8 pages, 2011.

[21] C. Sánchez-López, E. Martínez-Romero, and E. Tlelo-Cuautle, "Symbolic analysis of OTRAs-based circuits," Journal of Applied Research and Technology, vol. 9, no. 1, pp. 69-80, 2011.

[22] C. Sánchez-López, F. V. Fernández, E. Tlelo-Cuautle, and S. X. D. Tan, "Pathological element-based active device models 
and their application to symbolic analysis," IEEE Transactions on Circuits and Systems I: Regular Papers, vol. 58, no. 6, pp. 1382-1395, 2011.

[23] E. Tlelo-Cuautle, C. Sánchez-López, and D. Moro-Frías, "Symbolic analysis of (MO)(I)CCI(II)(III)-based analog circuits," International Journal of Circuit Theory and Applications, vol. 38, no. 6, pp. 649-659, 2010. 

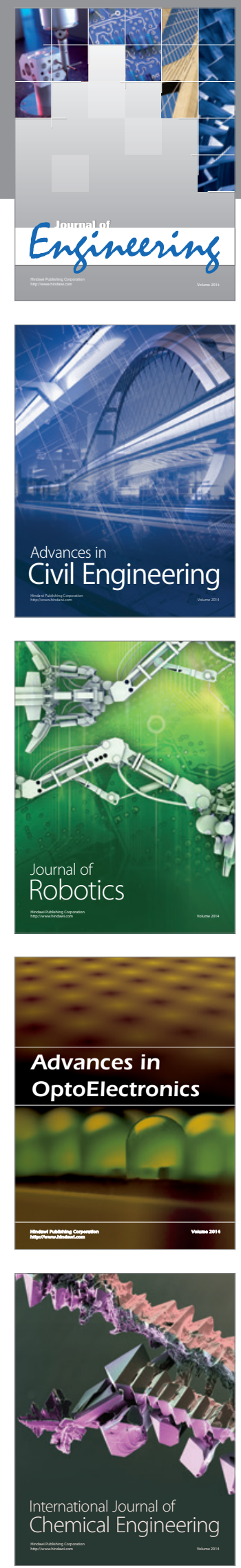

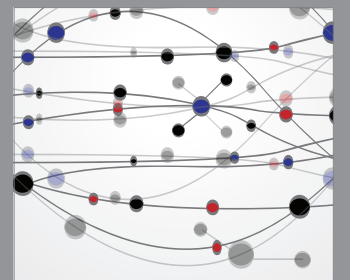

The Scientific World Journal
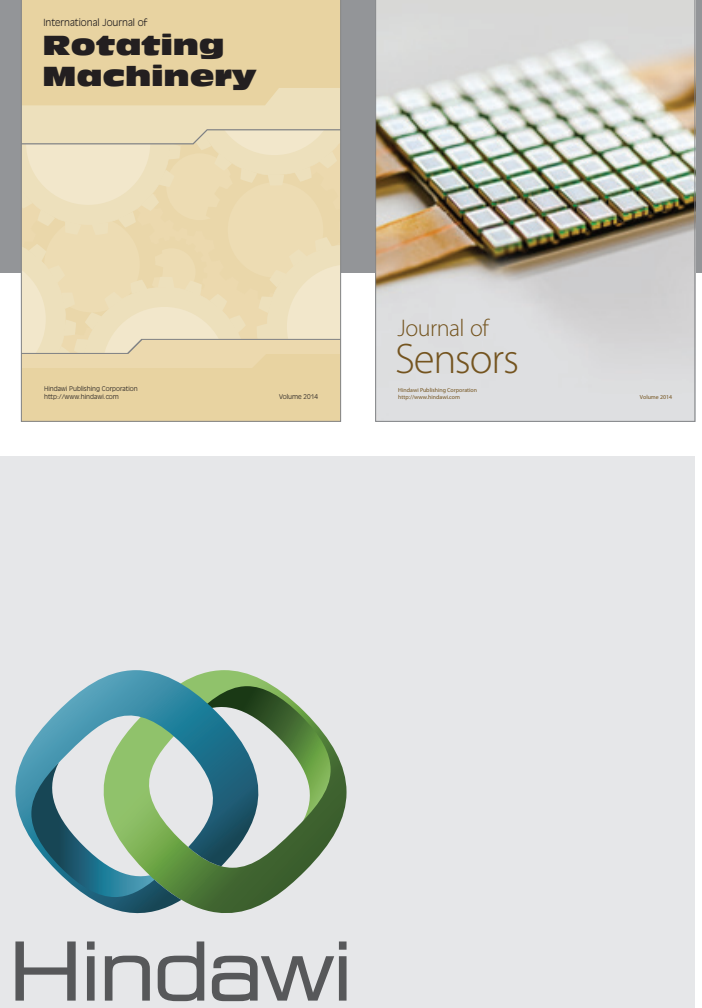

Submit your manuscripts at http://www.hindawi.com
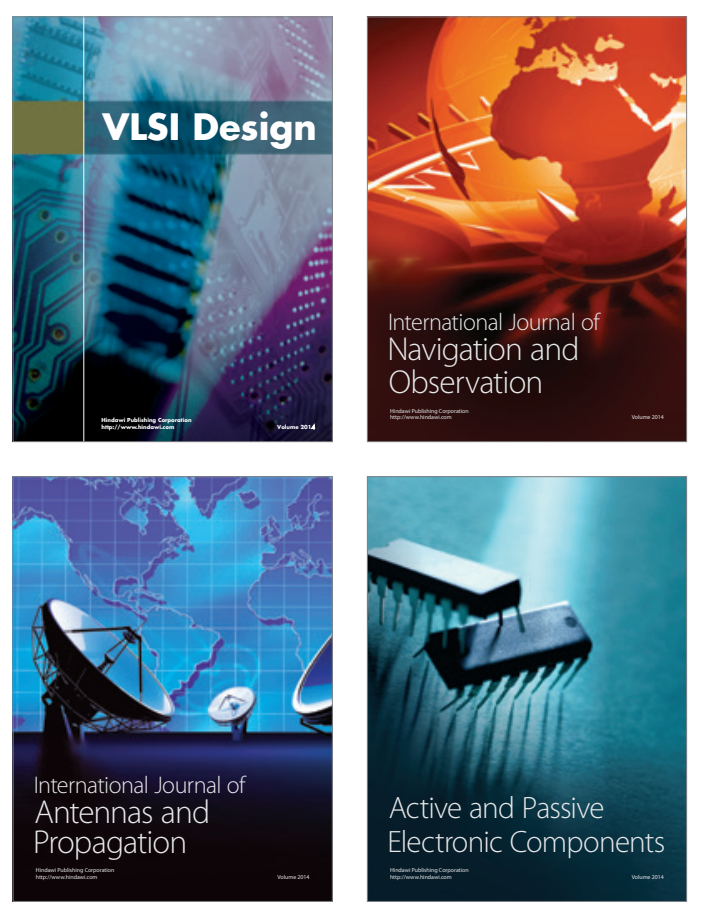
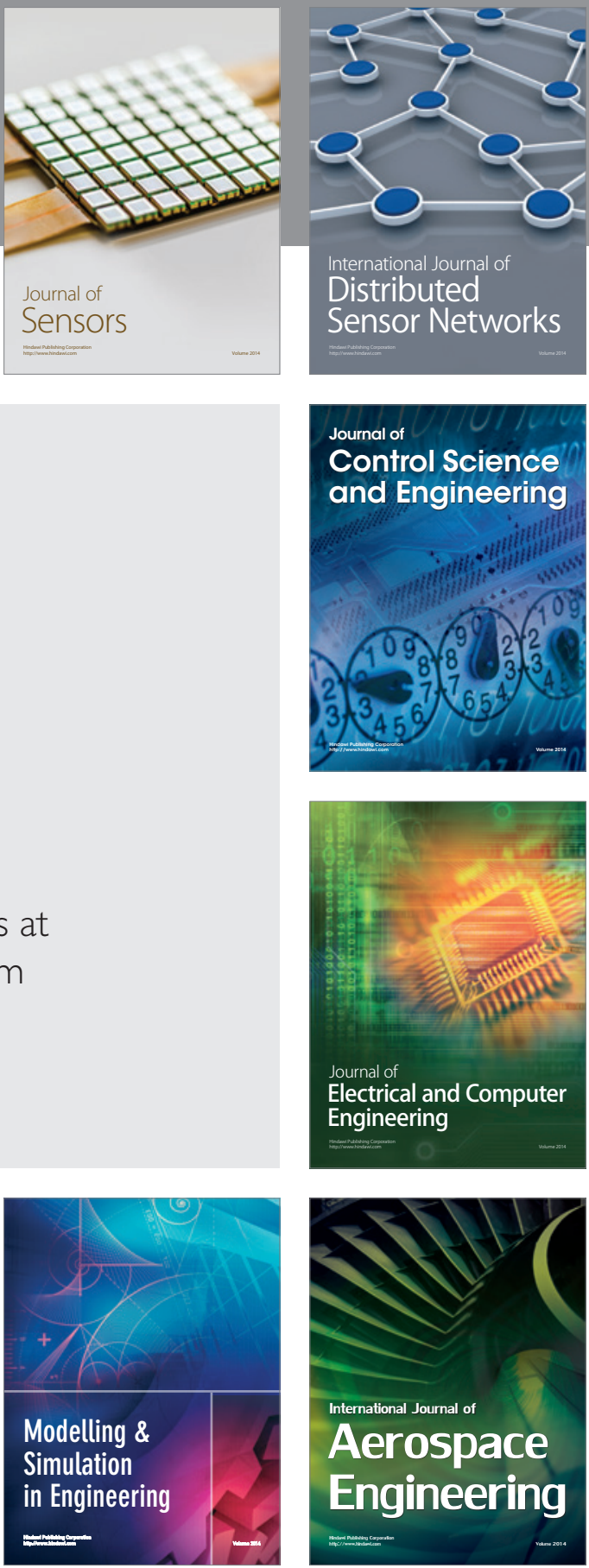

Journal of

Control Science

and Engineering
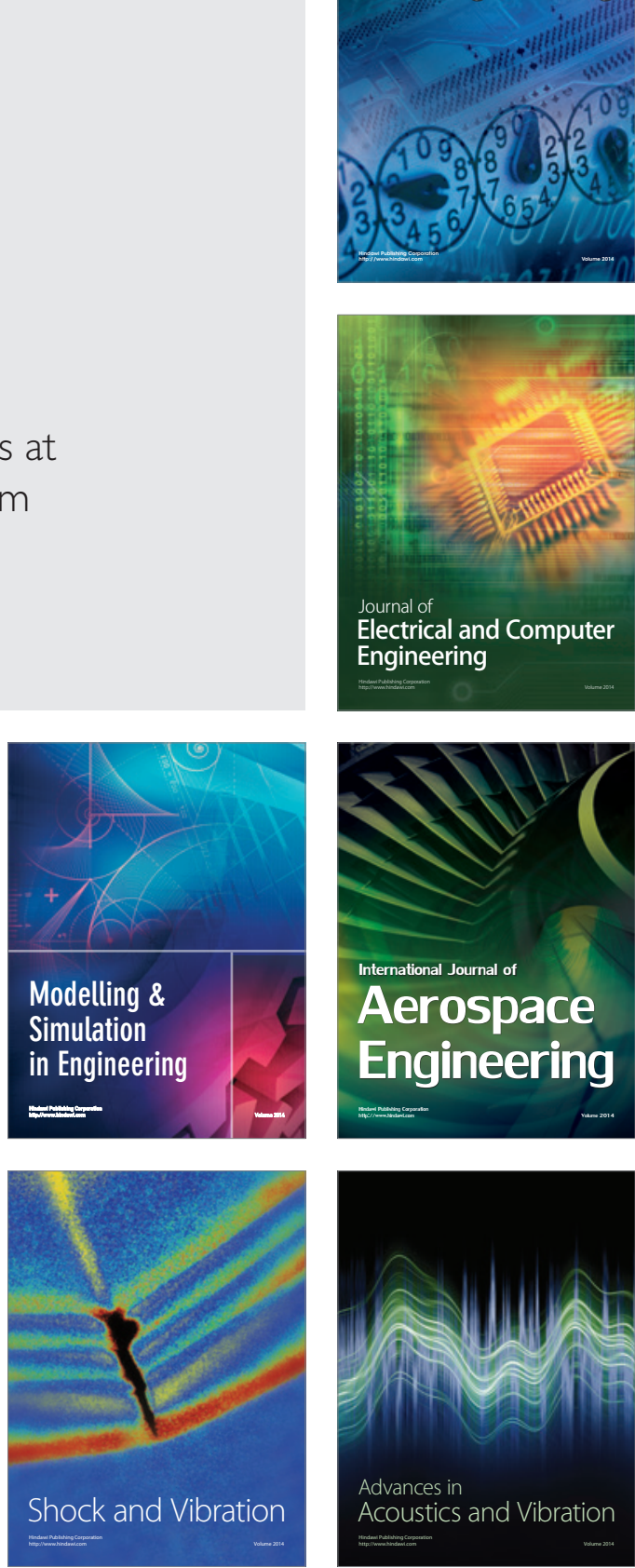